\title{
Microbially-mediated redox processes for sustainable treatment of micropollutants in water
}

\author{
MAGGIE L WHITE, JAMES J N KITSON AND ANKE \\ NEUMANN
}

Newcastle University

Presenting Author: m.l.white@newcastle.ac.uk

Water and wastewater treatment are essential for ensuring environmental and human health globally. However, the incomplete removal of emerging organic micropollutants during conventional wastewater treatment processes is of concern. Encouragingly, advanced oxidation processes (AOPs), which rely mainly on the production of reactive oxidizing species such as hydroxyl radicals ( $\mathrm{OH}$ radicals), have been successful in treating micropollutants. Current AOPs are energetically and chemically expensive with a high-carbon footprint and low-cost and low-input alternatives are required. Recent studies show that $\mathrm{OH}$ radicals can also be effectively generated and degrade micropollutants in the presence of natural iron-bearing clay minerals, e.g. nontronite. Currently it is unknown whether and how these redox-active clay minerals could be used to develop a novel, sustainable and low-carbon footprint AOP. This study aims to address this knowledge and suggests exploiting the interaction of Fe-bearing clay minerals with microbial communities in-situ to perform sustained and effective treatment of micropollutants in wastewaters over time.

To prove the concept for our novel sustainable AOP, we have been using laboratory mesocosm experiments. The columns contain the Fe-bearing clay mineral nontronite (NAu-2) and were seeded with a wild microbial community to stimulate clay mineral iron reduction. Synthetic wastewater containing oxygen and model micropollutants (benzoic acid and phenol) will be used to initiate the production of $\mathrm{OH}$ radicals and to monitor the extent and kinetics of micropollutant degradation. Using molecular microbiological methods, we are assessing the effect of the oxidative treatment on the microbial community, which will be complemented with investigations of (clay) mineralogical changes (XRD, Mössbauer spectroscopy, ATR-FTIR). Current experiments will yield time-series data sets to establish the number of clay mineral iron reduction and oxidative treatment cycles possible under realistic environmental conditions. 